\title{
Supplementary Information: A Critical
}

\section{Comparison of Biomembrane Force Fields: \\ Structure and Dynamics of Model DMPC, POPC, and POPE Bilayers}

Kristyna Pluhackova, ${ }^{*}{ }^{\dagger}$ Sonja A. Kirsch, ${ }^{\dagger}$ Jing Han, ${ }^{\dagger}$ Liping Sun ${ }^{\dagger}$ Zhenyan $^{2}$ Jiang, ${ }^{\dagger}$ Tobias Unruh, ${ }^{\ddagger}$ and Rainer A. Böckmann ${ }^{*, \dagger}$

$\dagger$ Computational Biology, Department of Biology, Friedrich-Alexander-University of Erlangen-Nürnberg, Staudtstr. 5, 91058 Erlangen

$\ddagger$ Lehrstuhl für Kristallografie und Strukturphysik, Department Physik,

Friedrich-Alexander-University of Erlangen-Nürnberg, Staudtstr. 3, 91058 Erlangen

E-mail: kristyna.pluhackova@fau.de; rainer.boeckmann@fau.de 
Table S1: Volumes of individual water molecule (in $\mathrm{nm}^{3}$ ) simulated using force field specific parameter file (mdp files in GROMACS) calculated out of $4 \mathrm{~ns}$ simulations containing 14572 water molecules.

\begin{tabular}{l|c|c|c|c}
\hline Force field & water model & $\mathbf{3 2 0 ~ K}$ & $\mathbf{3 0 0 ~ K}$ & $\mathbf{3 0 8 ~ K}$ \\
\hline CHARMM36 & TIPS3P $^{1}$ & 0.03020 & 0.02966 & 0.02988 \\
Slipids & TIP3P $^{2}$ & 0.03097 & 0.03038 & 0.03061 \\
Lipid14 & TIP3P $^{2}$ & 0.03097 & 0.03039 & 0.03061 \\
GROMOS54a7 & SPC $^{3}$ & 0.03125 & 0.03074 & 0.03093 \\
\hline
\end{tabular}



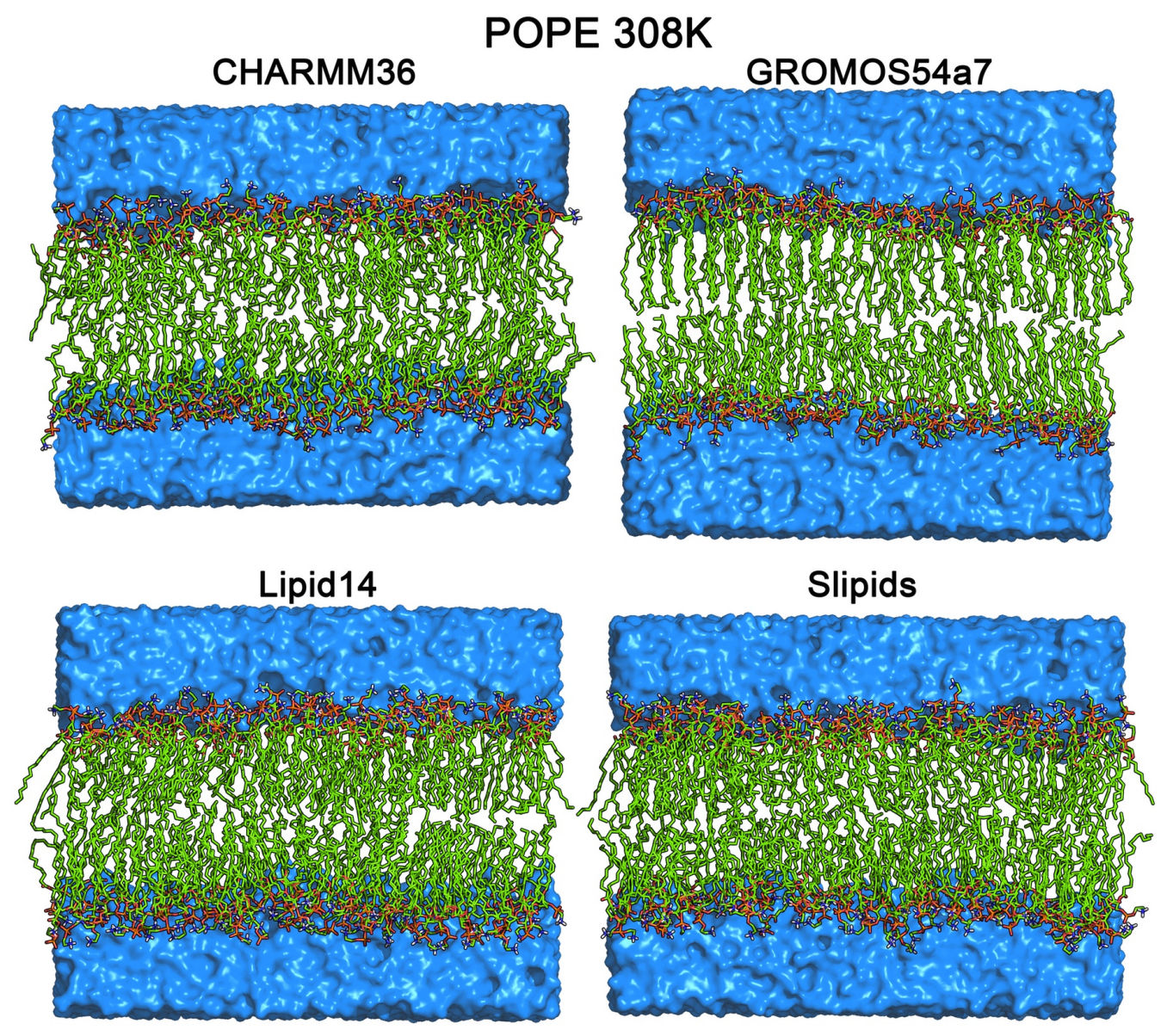

Figure S1: POPE simulation systems after $200 \mathrm{~ns}$ simulation time at $300 \mathrm{~K}$. Only GROMOS54a7 which is partially in a gel phase doesn't exhibit a proper fluid phase. Hydrogen atoms were omitted for clarity. Water is shown as blue surface, DMPC as atom-color-coded sticks (carbon in green, oxygen in red, nitrogen in blue, and phosphorus in orange). 


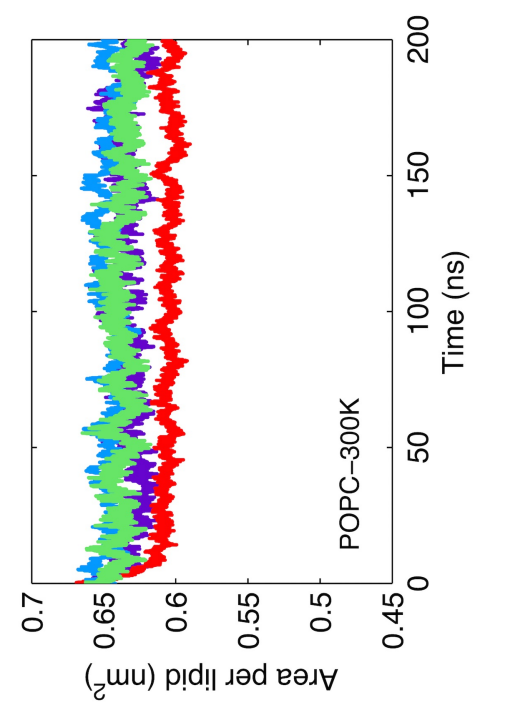

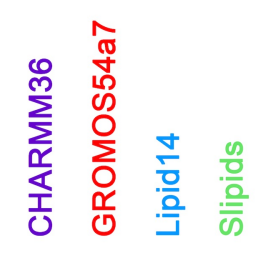

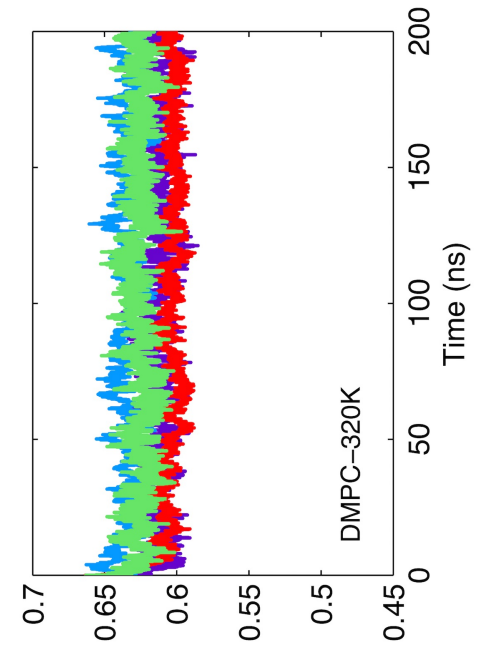

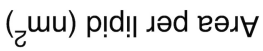
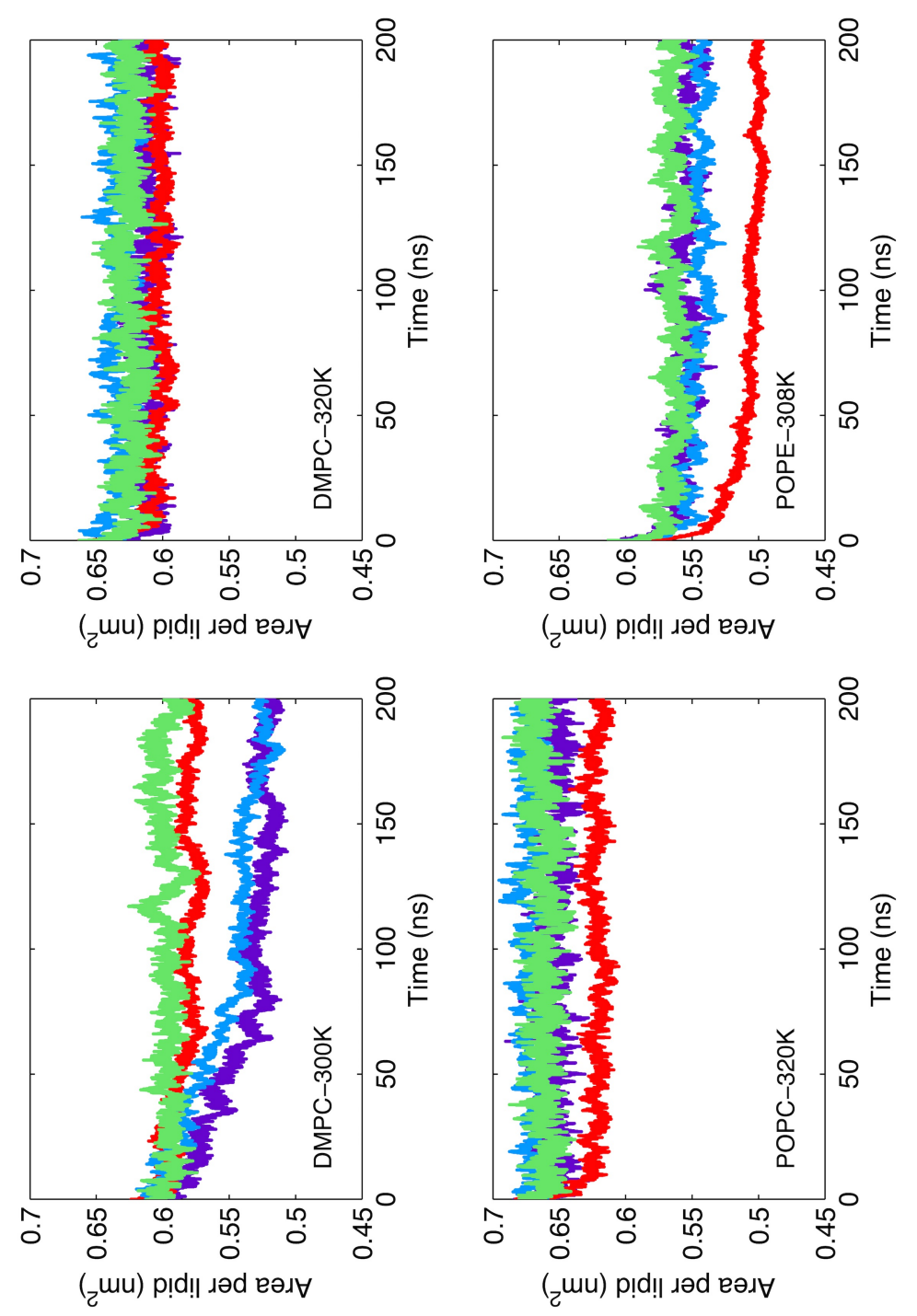

Figure S2: Evolution of the area per lipid over time. 


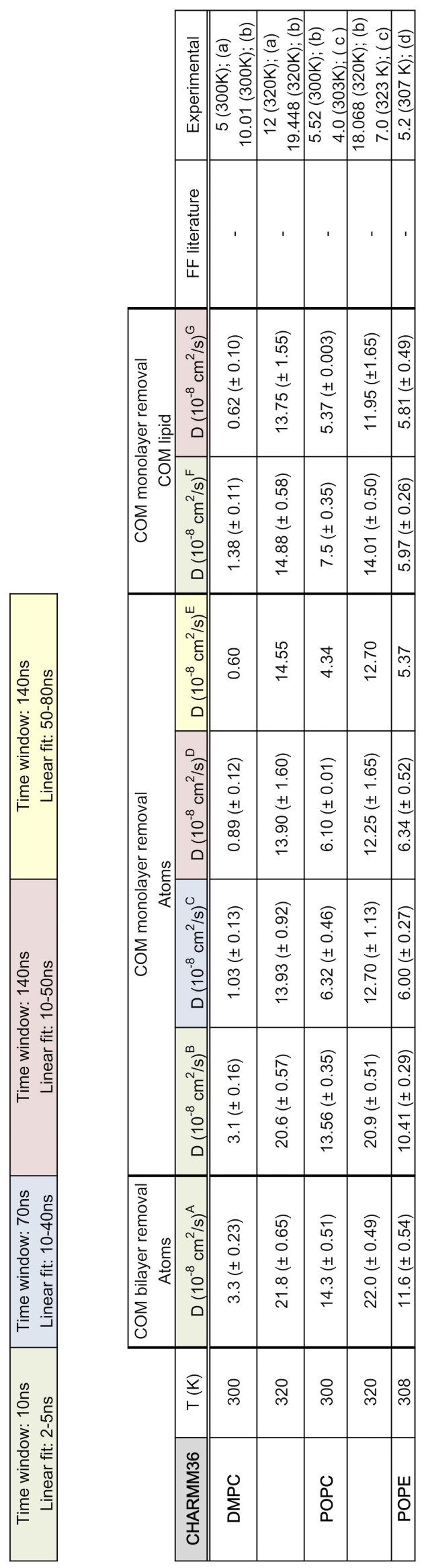

Figure S3: Diffusion coefficients calculated in different ways. In each simulation the first $60 \mathrm{~ns}$ were excluded for equilibration. The center of mass $(\mathrm{COM})$ motion of the whole bilayer (A) or separately of each monolayer (B-G) was removed. The mean square displacement (MSD) was calculated for lipid atoms (A$\mathrm{E}$ ) or for the lipid center of masses (FG). In green columns (A, B, F), the diffusion was calculated by splitting the simulations in $10 \mathrm{~ns}$ windows (linear fit on 2-5 ns). In blue columns (C), the simulations were split in $70 \mathrm{~ns}$ time windows (linear fitting from 10 to $40 \mathrm{~ns}$ ). In red $(\mathrm{D}, \mathrm{G})$ and yellow (E) columns, the diffusion was calculated from the whole 60 $200 \mathrm{~ns}$ simulations with linear fitting on $10-50 \mathrm{~ns}$ and $50-80 \mathrm{~ns}$, respectively. The diffusion coefficient was calculated separately for each monolayer and averaged over all atoms/molecules and over time. Experimental findings were taken from (a) Almeida et al. (1992), ${ }^{4}$ (b) Filippov et al. (2003), ${ }^{5}$ (c) Köchy et al. (1993), ${ }^{6}$ and (d) Jin et al. (1999; $78 \mathrm{~mol} \% \mathrm{PE}){ }^{7}$ The difference between the diffusion coefficients, calculated with the aid of the lipid atoms, and a removal of the bilayer (A) and monolayer (B) COM motion, is rather small. The same holds true for the diffusion coefficients obtained by lipid atoms (D) and lipid COM (G) for the whole 140 ns interval. Overall, the diffusion coefficients calculated over larger time windows exhibit smaller values compared to time windows of small duration. 

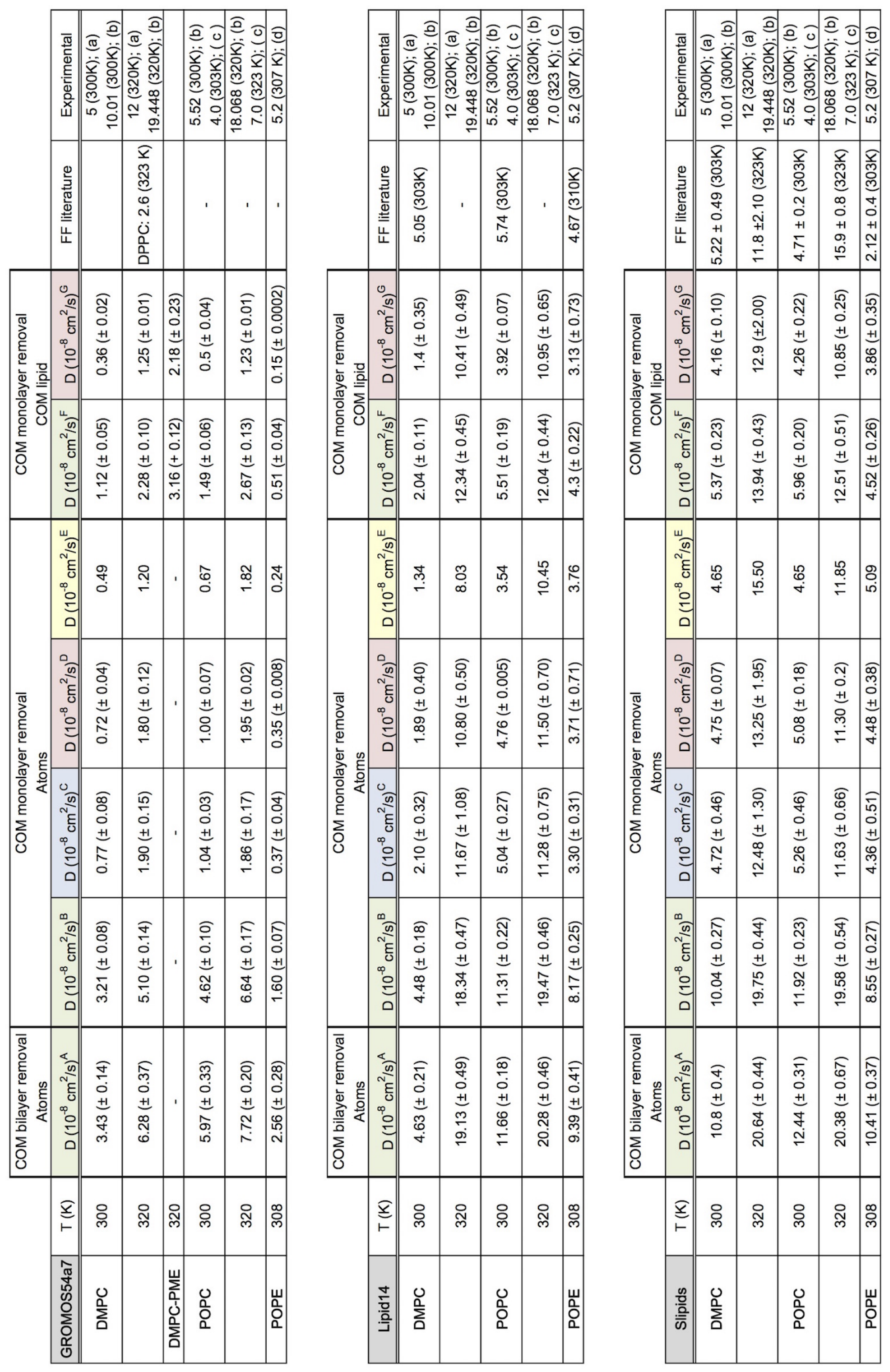
Table S2: Detailed results of lipid chain protrusion analysis. Analysis performed on 60-200 ns of simulations. Protrusions recorded at a single frame (10 ps) were excluded from the analysis. The value in paranthesis by median time denotes the interquartile range. Protrusion efficiency is a product of protrusion probability and the median time of a protrusion event.

\begin{tabular}{|c|c|c|c|c|c|c|}
\hline $\begin{array}{c}\text { Simulated } \\
\text { System }\end{array}$ & layer & $\begin{array}{c}\text { number of } \\
\text { protrusions }\end{array}$ & $\begin{array}{c}\text { probability } \\
\text { per lipid } \\
{[1 / \mu \mathrm{s}]}\end{array}$ & $\begin{array}{c}\text { median time } \\
\text { (IQR) } \\
\text { [ns] }\end{array}$ & $\begin{array}{c}\text { maximal } \\
\text { time } \\
{[\mathrm{ns}]}\end{array}$ & $\begin{array}{l}\text { protrusion } \\
\text { efficiency }\end{array}$ \\
\hline \multicolumn{7}{|l|}{ CHARMM36 } \\
\hline DMPC & lower & 33 & 1.228 & $0.38(1.68)$ & 8.09 & 0.467 \\
\hline $300 \mathrm{~K}$ & upper & 35 & 1.302 & $0.46(3.23)$ & 12.11 & 0.599 \\
\hline DMPC & lower & 77 & 2.865 & $0.17(0.50)$ & 2.81 & 0.487 \\
\hline $320 \mathrm{~K}$ & upper & 74 & 2.753 & $0.12(0.37)$ & 3.62 & 0.317 \\
\hline POPC & lower & 44 & 1.637 & $0.41(1.19)$ & 3.48 & 0.663 \\
\hline $300 \mathrm{~K}$ & upper & 46 & 1.711 & $0.34(0.83)$ & 2.94 & 0.582 \\
\hline POPC & lower & 72 & 2.679 & $0.18(0.43)$ & 2.43 & 0.482 \\
\hline $320 \mathrm{~K}$ & upper & 86 & 3.199 & $0.20(0.41)$ & 1.76 & 0.624 \\
\hline POPE & lower & 7 & 0.260 & $0.04(0.99)$ & 2.53 & 0.010 \\
\hline $308 \mathrm{~K}$ & upper & 12 & 0.446 & $0.13(0.32)$ & 2.61 & 0.056 \\
\hline \multicolumn{7}{|l|}{ Slipids } \\
\hline DMPC & lower & 65 & 2.418 & $0.27(0.69)$ & 4.86 & 0.653 \\
\hline $300 \mathrm{~K}$ & upper & 59 & 2.195 & $0.29(0.90)$ & 5.62 & 0.637 \\
\hline DMPC & lower & 146 & 5.432 & $0.24(0.55)$ & 3.14 & 1.304 \\
\hline $320 \mathrm{~K}$ & upper & 121 & 4.501 & $0.24(0.55)$ & 2.28 & 1.080 \\
\hline POPC & lower & 66 & 2.455 & $0.33(0.83)$ & 4.95 & 0.798 \\
\hline $300 \mathrm{~K}$ & upper & 71 & 2.641 & $0.25(1.11)$ & 3.52 & 0.660 \\
\hline POPC & lower & 162 & 6.027 & $0.17(0.60)$ & 3.32 & 0.994 \\
\hline $320 \mathrm{~K}$ & upper & 152 & 5.655 & $0.13(0.37)$ & 2.3 & 0.707 \\
\hline POPE & lower & 30 & 1.116 & $0.34(0.82)$ & 4.08 & 0.379 \\
\hline $308 \mathrm{~K}$ & upper & 33 & 1.228 & $0.24(0.75)$ & 2.84 & 0.295 \\
\hline \multicolumn{7}{|l|}{ Lipid14 } \\
\hline DMPC & lower & 326 & 12.128 & $0.40(1.25)$ & 20.85 & 4.791 \\
\hline $300 \mathrm{~K}$ & upper & 209 & 7.775 & $0.57(1.30)$ & 22.43 & 4.432 \\
\hline DMPC & lower & 362 & 13.467 & $0.16(0.51)$ & 3.09 & 2.155 \\
\hline $320 \mathrm{~K}$ & upper & 367 & 13.653 & $0.18(0.55)$ & 3.83 & 2.458 \\
\hline POPC & lower & 249 & 9.263 & $0.24(0.63)$ & 4.21 & 2.223 \\
\hline $300 \mathrm{~K}$ & upper & 267 & 9.933 & $0.26(0.70)$ & 4.47 & 2.583 \\
\hline POPC & lower & 426 & 15.848 & $0.17(0.45)$ & 4.55 & 2.694 \\
\hline $320 \mathrm{~K}$ & upper & 424 & 15.774 & $0.19(0.49)$ & 3.58 & 2.997 \\
\hline POPE & lower & 217 & 8.073 & $0.25(0.78)$ & 5.32 & 2.018 \\
\hline $308 \mathrm{~K}$ & upper & 186 & 6.920 & $0.33(0.72)$ & 4.14 & 2.249 \\
\hline \multicolumn{7}{|l|}{ GROMOS54a7 } \\
\hline DMPC & lower & 25 & 0.930 & $0.89(1.74)$ & 5.04 & 0.487 \\
\hline $300 \mathrm{~K}$ & upper & 30 & 1.116 & $0.65(1.51)$ & 14.82 & 0.487 \\
\hline DMPC & lower & 45 & 1.674 & $0.34(1.28)$ & 5.75 & 0.487 \\
\hline $320 \mathrm{~K}$ & upper & 58 & 2.158 & $0.47(1.53)$ & 4.68 & 0.487 \\
\hline POPC & lower & 22 & 0.818 & $0.50(1.79)$ & 6.48 & 0.487 \\
\hline $300 \mathrm{~K}$ & upper & 28 & 1.042 & $0.68(1.70)$ & 3.96 & 0.487 \\
\hline POPC & lower & 47 & 1.749 & $0.21(1.15)$ & 4.1 & 0.487 \\
\hline $320 \mathrm{~K}$ & upper & 51 & 1.897 & $0.30(1.36)$ & 16.45 & 0.487 \\
\hline POPE & lower & 1 & 0.037 & $1.33(0.00)$ & 1.33 & 0.049 \\
\hline $308 \mathrm{~K}$ & upper & 1 & 0.037 & $0.02(0.00)$ & 0.02 & 0.001 \\
\hline
\end{tabular}



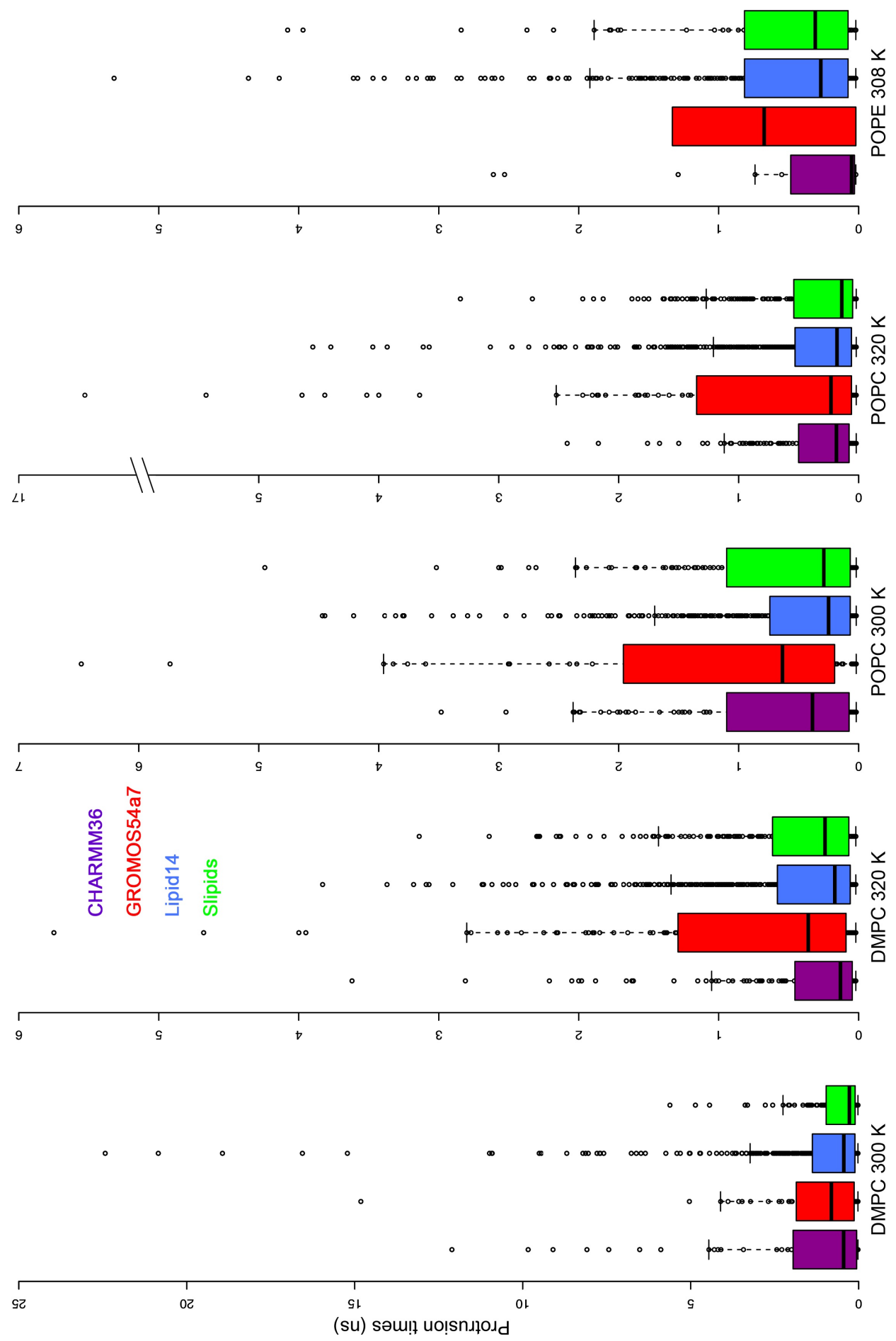

Figure S4: Box plots of lipid protrusion times for systems under study. CHARMM36 is colored in purple, GROMOS54a7 in red, Lipid14 in blue, and Slipids in green. Horizontal top and bottom lines of each box define the first and third quartiles, the thick line inside the box is the median. The whiskers (horizontal lines outside the box) determine the last data point that is no more away than 1.5 times the interquartile range (box length) from the box. Box plots were generated with RStudio. ${ }^{8}$ 


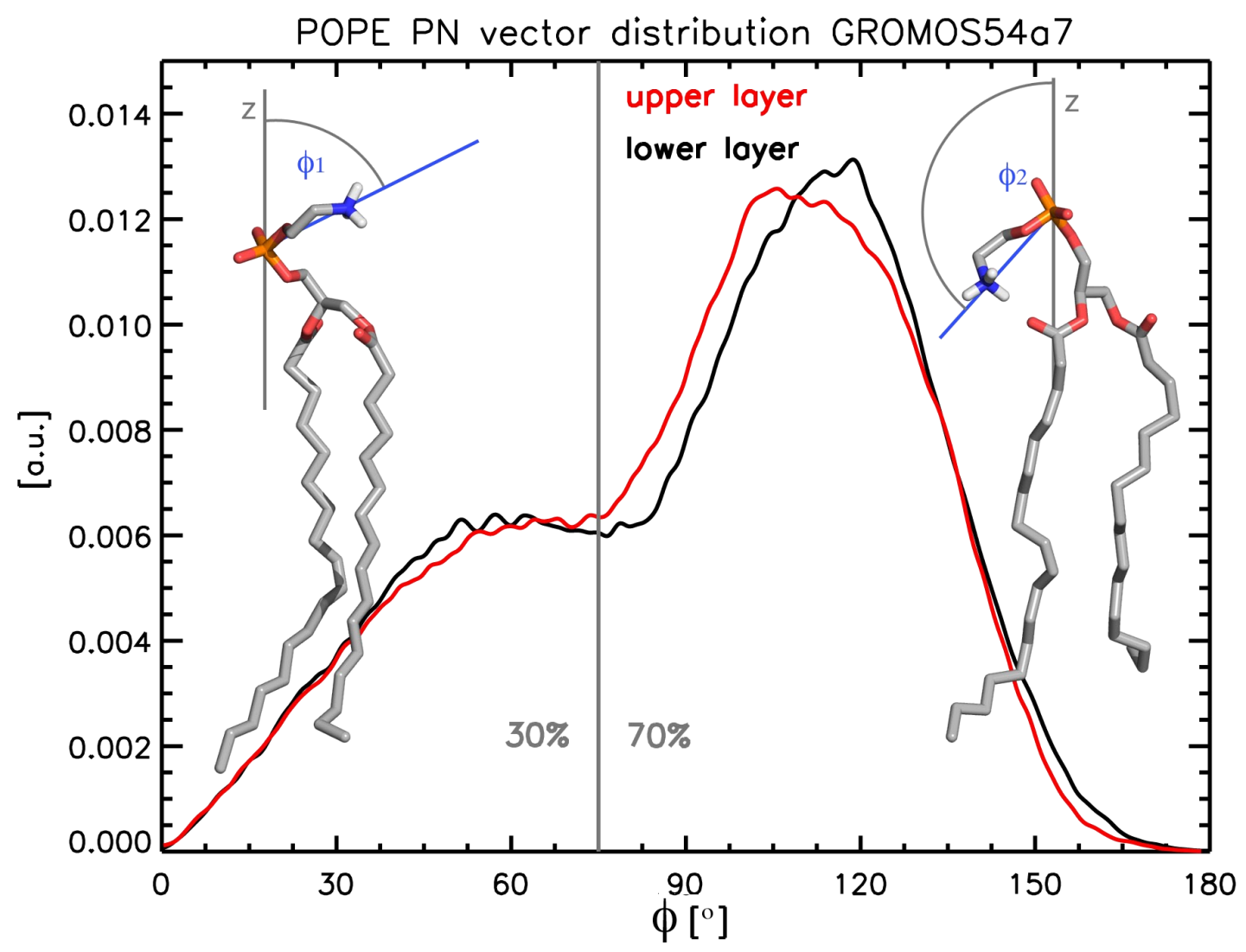

Figure S5: Distribution of PN vectors relative to the membrane normal for the POPE gel phase obtained by the GROMOS54a7 force field. The population of lipids with the PN vector smaller than $75^{\circ}$ (an example lipid shown on the left) corresponds to $30 \%$, the population of lipids with the PN vector larger than $75^{\circ}$ (an example lipid shown on the right) makes up $70 \%$. 


\section{Calculation of order parameters of unsaturated carbons}

Using the GROMACS's tool g_order to calculate the order parameters around unsaturated carbons succeeds by following following workflow. The test case is a lipid containing one saturated chain (palmitoyl carbons named C2A - C2P) and one monounsaturated (oleoyl, carbons $\mathrm{C} 1 \mathrm{~A}-\mathrm{C} 1 \mathrm{R})$ chain.

\section{Step 1. Calculation of standard saturated order parameters for all carbons.}

First index files have to be created for each chain individually containing only carbons for which order parameters should be calculated. Please note, that using g_order no order parameter value can be calculated for the first and the last carbon in the chain.

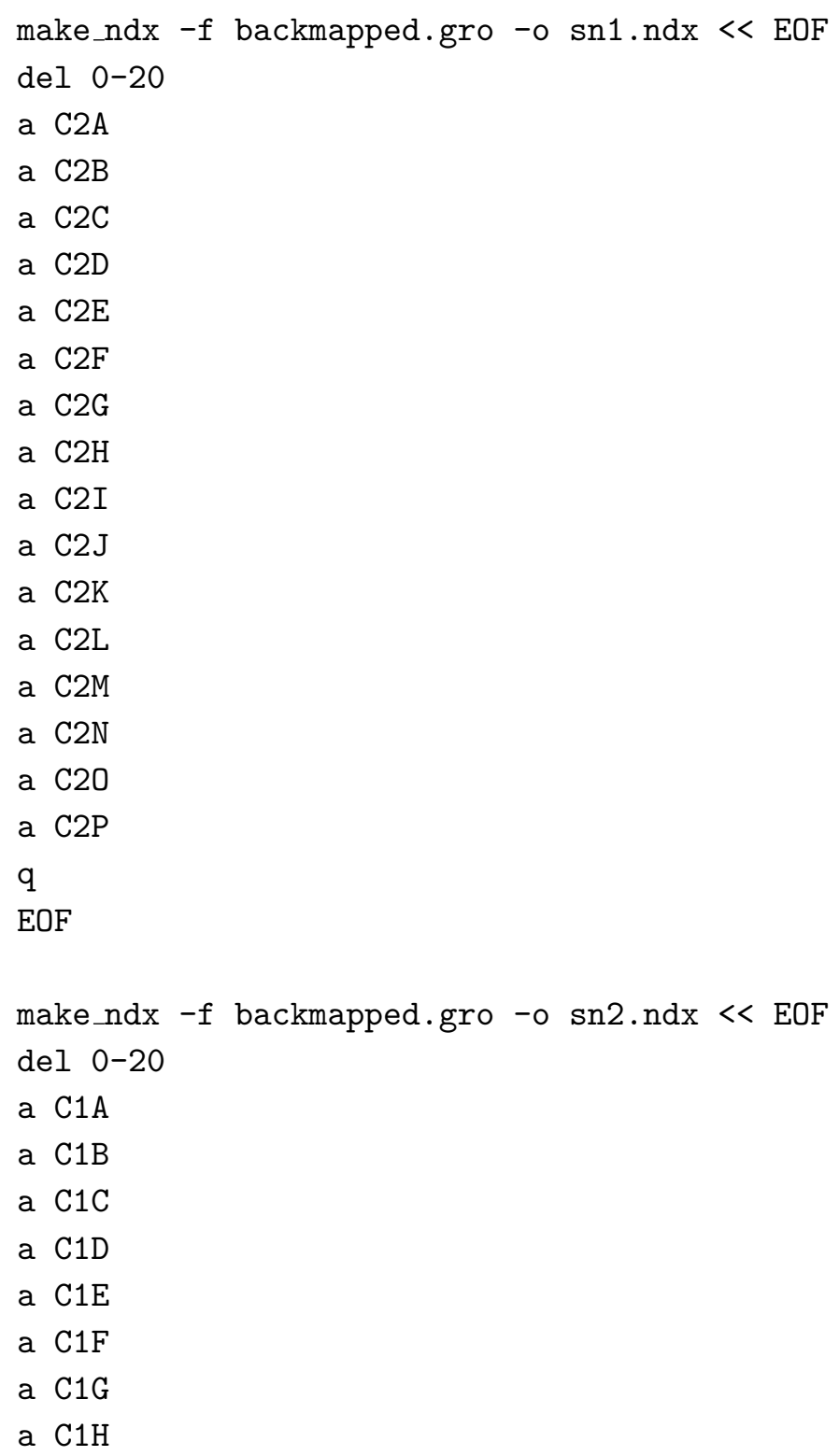



a C1I
a $\mathrm{C} 1 \mathrm{~J}$
a $\mathrm{C} 1 \mathrm{~K}$
a $\mathrm{C} 1 \mathrm{~L}$
a $\mathrm{C} 1 \mathrm{M}$
a $\mathrm{C} 1 \mathrm{~N}$
a $\mathrm{C} 10$
a C1P
a $\mathrm{C} 1 \mathrm{Q}$
a C1R
q
EOF

Next g_order is used to calculate the order parameters for each chain.

g_order -s topol.tpr -f traj.xtc -n sn1.ndx -d z -od deuter_sn1.xvg

g_order -s topol.tpr -f traj.xtc -n sn2.ndx -d z -od deuter_sn2.xvg

\section{Step 2. Calculation of order parameters for unsaturated carbons}

The double bond is found between atoms C1I and C1J. Calculation of the order parameter for atom $\mathrm{C} 1 \mathrm{~J}$ ( - C1I = C1J - C1K - ) succeeds following

make ndx $-f$ backmapped.gro -0 sn2-C1J.ndx $<$ EOF

del $0-20$

a $\mathrm{C} 1 \mathrm{I}$

a $\mathrm{C} 1 \mathrm{~J}$

a $\mathrm{C} 1 \mathrm{~K}$

q

EOF

g_order -unsat -f traj.xtc -s topol.tpr -n sn2-C1J.ndx -d z

-od deuter_C1J.xvg

The order parameter for atom C1I is calculated following the opposite direction ( - C1J $=\mathrm{C} 1 \mathrm{I}-\mathrm{C} 1 \mathrm{H}-$ ).

make $n d x-f$ backmapped.gro -o sn2-C1I.ndx $<<$ EOF

del $0-20$

a $\mathrm{C} 1 \mathrm{~J}$

a $\mathrm{C} 1 \mathrm{I}$

a $\mathrm{C} 1 \mathrm{H}$

q

EOF 
g_order -unsat -f traj.xtc -s topol.tpr -n sn2-C1I.ndx -d z

-od deuter_C1I.xvg

\section{Step 3. Combining results}

In the last step the order parameters in the file deuter_sn2.xvg of atoms C1J and C1I are replaced by results obtained by calculation using the option -unsat and proper atom order.

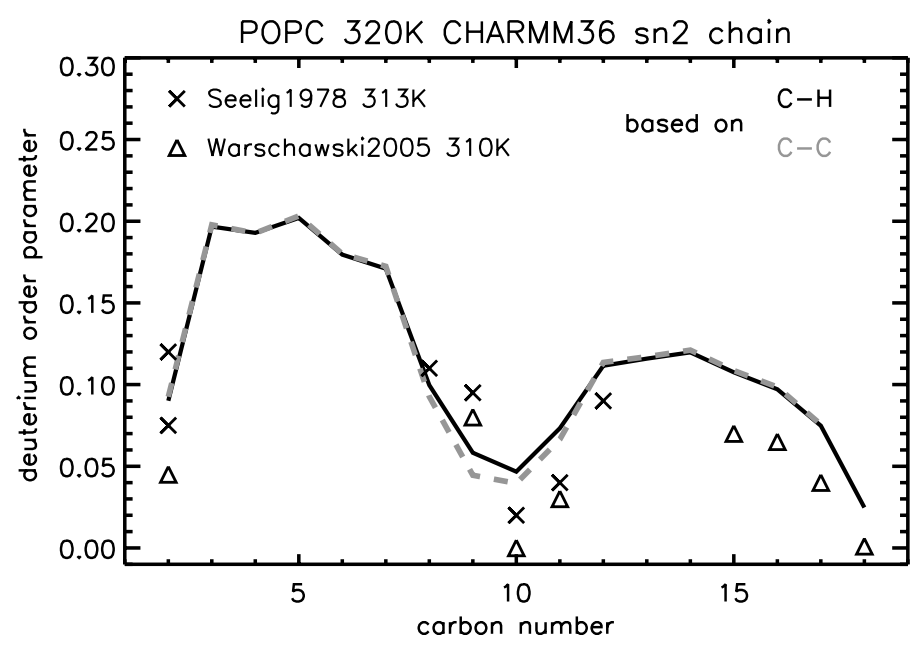

Figure S6: Deuterium order parameters of the unsaturated sn2 chain of POPC simulated by CHARMM36 at $320 \mathrm{~K}$ calculated based on C-H orientation (black) or C-C orientation (grey). The latter method is applied in GROMACS' tool g_order. The spread of the experimental data (crosses and triangles) is larger than the difference between the two theoretical methods.

\section{References}

(1) MacKerell, A. D.; Bashford, D.; Bellott,; Dunbrack, R. L.; Evanseck, J. D.; Field, M. J.; Fischer, S.; Gao, J.; Guo, H.; Ha, S. et al. All-atom empirical potential for molecular modeling and dynamics studies of proteins. J. Phys. Chem. B 1998, 102, 3586-3616.

(2) Sun, Y.; Kollman, P. A. Hydrophobic solvation of methane and nonbond parameters of the TIP3P water model. J. Comput. Chem. 1995, 16, 1164-1169.

(3) Berendsen, H. J. C.; Postma, J. P. M.; van Gunsteren, W. F.; Hermans, J. Interaction models for water in relation to protein hydration. Intermolecular Forces 1981, 331-342.

(4) Almeida, P. F.; Vaz, W. L.; Thompson, T. Lateral diffusion in the liquid phases of dimyristoylphosphatidylcholine/cholesterol lipid bilayers: a free volume analysis. Biochemistry 1992, 31, 6739-6747. 
(5) Filippov, A.; Orädd, G.; Lindblom, G. Influence of cholesterol and water content on phospholipid lateral diffusion in bilayers. Langmuir 2003, 19, 6397-6400.

(6) Köchy, T.; Bayerl, T. M. Lateral diffusion coefficients of phospholipids in spherical bilayers on a solid support measured by ${ }^{2} \mathrm{H}$-nuclear-magnetic-resonance relaxation. Phys. Rev. E 1993, 47, 2109.

(7) Jin, A. J.; Edidin, M.; Nossal, R.; Gershfeld, N. A singular state of membrane lipids at cell growth temperatures. Biochemistry 1999, 38, 13275-13278.

(8) RStudio Team, RStudio: Integrated Development Environment for R. RStudio, Inc.: Boston, MA, 2015. 